\title{
OCORRÊNCIA DE PLÁSTICO NO CONTEÚDO ESTOMACAL DO CAMARÃO-DE-PROFUNDIDADE ARISTAEOPSIS EDWARDSIANA (CRUSTACEA, ARISTEIDAE).
}

\author{
GABRIELA AMARAL DE REZENDE ${ }^{1}$; PAULO RICARDO PEZZUTO²; FERNANDO D'INCAO ${ }^{1}$ \\ ${ }^{1}$ Universidade Federal do Rio Grande - Laboratório de Crustáceos Decápodos, Rio Grande - RS - Brasil. CEP: 96201-900 - Caixa postal: 474. \\ gab_rezende@hotmail.com \\ ${ }^{2}$ Centro de Educação Superior de Ciências Tecnológicas da Terra e do Mar; CTTMar/UNIVALI - pezzuto@univali.br
}

\section{RESUMO}

Aristaeopsis edwardsiana é um camarão de profundidade registrado em profundidades que variam de $400-900 \mathrm{~m}$. Ao examinar a dieta de Aristaeopsis edwardsiana foram encontrados resíduos plásticos no estômago de três indivíduos. Este parece ser o primeiro registro de ingestão de resíduos plástico por esta espécie.

PALAVRAS CHAVE: resíduos plásticos, dieta, Aristaeopsis edwardsiana.

\section{ABSTRACT}

Ocurrence of plastic debris on stomachs contents of the deep-sea shrimp Aristaeopsis edwardsiana (Crustacea, Aristeidae)

Aristaeopsis edwardsiana is a deep-sea shrimp registered at depths from $400-900 \mathrm{~m}$. By examining the diet of Aristaeopsis edwardsiana was found that three individuals had plastic debris on their stomachs. This appeared to be the first record of ingestion of plastic debris by this species.

KEYWORDS: plastic debris; diet; Aristaeopsis edwardsiana.

$\mathrm{Na}$ costa brasileira, os camarões da família Aristaeidae compreendem um dos mais valiosos recursos de mar profundo, em que Aristaeopsis edwardsiana é a principal espécie capturada (Pezzuto et al. 2006). Está espécie é predominante de profundidade, e ocorre frequentemente em profundidades entre 400 e 900 m (D'Incao 1998). Aristeídeos são extremamente abundantes na coluna d'água e possuem um importante papel na cadeia alimentar marinha, são predadores ativos e presas de muitos peixes (Kapiris et al. 2010).

A presença de lixo plástico é comum em áreas costeiras próximas a centros urbanos e em regiões afastadas da presença urbana (Convey et al. 2002). A descarga de lixo plástico no ambiente marinho pode ocorrer acidentalmente ou deliberadamente, tanto por atividades humanas nestes ambientes como pesca, navios mercantes, cruzeiros, e barcos recreacionais, ou por descarga de rios ou drenagem realizada pelos ventos (Gillian et al. 1992, Laist et al. 1999). O lixo pode ser levado por milhares de milhas do seu ponto de entrada no mar e viajar pelos oceanos por muitos anos (Ribic \& Gani 1996, Laist 1999).

O lixo marinho afeta pelo menos 267 espécies de animais marinhos, incluindo $86 \%$ das tartarugas marinhas, $44 \%$ das aves e $43 \%$ dos mamíferos marinhos e muitas espécies de peixes e crustáceos (Laist 1997). A ingestão de plástico pode causar vários efeitos deletérios na condição física e sobrevivência das espécies (Katsanevaskis 2008).
Thompson et al. (2004) conduziu experimentos usando o ANFÍPODES, e mostrou que a ingestão de plástico foi devido a confusão do plástico com a comida. Também, já foram observados filamentos de nylon no conteúdo estomacal de Nephrops uma lagosta de valor comercial capturada no Mar Adriático (Wieczorek et al. 1999). Contudo, estudos na ingestão de plástico por crustáceos ainda são limitados.

Este trabalho tem por objetivo documentar a ocorrência de lixo plástico no conteúdo estomacal do camarão de profundidade Aristaeopsis edwardsiana na Costa Sudeste do Brasil.

Ao examinar a dieta de 215 indivíduos de Aristaeopsis edwardsiana, foi encontrado lixo plástico em três indivíduos (duas fêmeas e um macho). Uma fêmea possuía $87,6 \mathrm{~mm}$ de comprimento de carapaça e foi capturada entre $25^{\circ} 25^{\prime} 03^{\prime \prime} \mathrm{S}$ e $44^{\circ} 36^{\prime} 35^{\prime \prime}$ W. A outra fêmea possuía $79,5 \mathrm{~mm}$ de comprimento de carapaça e a posição de captura era desconhecida. 0 macho possuía $52 \mathrm{~mm}$ de comprimento de carapaça e foi capturado entre $26^{\circ} 45^{\prime} 04^{\prime \prime} S$ e $46^{\circ} 20^{\prime} 41^{\prime \prime}$ W. Todos os indivíduos foram capturados pela pescaria de arrasto comercial de profundidade no Talude Continental da Costa de São Paulo, que ocorreu em 2005 entre as profundidades de 730-740m.

Os lixos plásticos encontrados no conteúdo estomacal de $A$. edwardsiana eram pretos, transparentes com verde, e azul translúcido (FIGURA 1). Em geral, pedaços de lixo plástico possuíam tamanho entre $0,05-0,7 \mathrm{~cm}$ (medidos pelo maior 


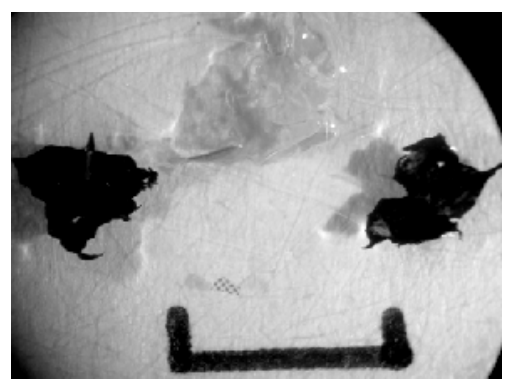

FIGURA 1 - Lixo plástico ingerido por Aristaeopsis edwardsiana. A espécie foi capturada no Talude Continental da Costa de São Paulo. Escala: $0,5 \mathrm{~cm}$.

Os estômagos dos três indivíduos estavam cheios, e outros itens foram encontrados no conteúdo estomacal (i.e, restos de peixes, sedimento e restos de crustáceos).

O plástico pode ser encontrado no mar em proporções semelhantes aos outros tipos de detritos, contudo possuem um grande uso, principalmente por marinheiros (Laist 1999). Além disso, devido ao seu peso leve e sua persistência no meio ambiente, o plástico é o mais comum dos detritos antropogênicos encontrados no mar (Laist et al. 1999).

Altas concentrações de detritos plástico são encontrados próximos a rotas marítimas (Gottfried et al. 1987), áreas de pesca, correntes de convergência e giros oceânicos (Pruter 1987). A presença de detritos também já foi registrada em fundos oceânicos profundos no Mar Mediterrâneo (Galil et al. 1995). Galgani et al. (1996), estudaram a distribuição e abundância de detritos marinhos no talude continental e planície abissal do noroeste do Mediterrâneo, eles observaram que a maioria dos detritos é encontrada nos cânyons e planície abissal, com grandes quantidades em profundidades maiores de $500 \mathrm{~m}$. A acumulação de detritos também está relacionada à fatores geomorfológicos, como a conformação do fundo oceânico, particularmente á existência de rochas, fendas e outras estruturas favoráveis a acumulação de detritos.

A ingestão de detritos plásticos pode reduzir a ingestão de alimento, o estímulo para alimentação, causar injúrias internas e morte por bloqueio do trato intestinal (Rothstein 1973, Azzarello \& Van-Vleet 1987, Ryan 1988, Zitko \& Hanlon 1991).
Outros efeitos deletérios incluem a diminuição do nível dos hormônios esteróides, atraso na ovulação e falha reprodutiva (Azzarello \& Van-Vleet 1987).

A análise dos conteúdos alimentares de Aristaeopsis edwardsiana mostra que a espécie se alimenta principalmente de crustáceos, peixes e cefalópodes (Rezende 2011). Ainda, considerando a pequena porcentagem de indivíduos analisados que apresentaram plástico em seu conteúdo (1,4\%) assume-se que a ingestão de plástico pela espécie tenha sido acidental.

Este é o primeiro registro da ingestão de plástico pelo camarão de profundidade Aristaeopsis edwardsiana na costa brasileira. Para crustáceos a ingestão de plástico e seus efeitos ainda não estão bem documentados.

\section{AGRADECIMENTOS}

Este estudo foi financiado pelo CNPq (Conselho Nacional de Desenvolvimento Científico e Tecnológico) e Governo Brasileiro (SEAP/PR/001/2003; SEAP/PR/078/2004; SEAP/PR/ 064/2005; SEAP/PR/027/2007). Agradecemos a equipe de observadores de bordo "Grupo de Estudos Pesqueiros" por disponibilizarem informações indispensáveis para este estudo.

\section{REFERÊNCIAS}

AZZARELLO, M.Y. \& VAN-VLEET, E.S.. 1987. Marine birds and plastic pollution. Mar. Ecol. Prog. Ser., 37: 295-303.

CONVEY, P.; BARNES, D.K.A \& MORTON, A.. 2002. Debris accumulation on oceanic island shores of the Scotia Arc, Antártica: a review. Polar Biol., 25(8):612-617.

D'INCAO, F.. 1998. The Brazilian species of the Family Aristeidae Wood-Mason (Crustacea:Decapoda). J. Nat. Hist., 32:1509- 
1518.

GALGANI, F.; SOUPLET, A. \& CADIOU, Y.. 1996. Accumulation of debris on the deep sea floor of the French Mediterranean Coast. Mar. Ecol. Prog. Ser 142: 225-234.

GALIL, B.S.; GOLIK, A. \& TUERKAY, M .. 1995. Litters at the bottom of the sea. A sea bed survey in the eastern Mediterranean Sea. Mar. Pollut. Bull.. 30(I): 22-24

GILLIGAN, M.R.; RANDAL, S.P.; RICHARDSON, J.P. \& KOZEL, T.R., 1992. Rates of Accumulation of Marine Debris in Chatham County, Georgia. Mar. Pollut. Bull 24(9), 436:441.

GOTTFRIED, J.M, VAUK, J.M, SCHREY E. 1987 Litter pollution from ships in the German Bight. Mar. Pollut. Bull. 18(6-B):316319

KAPIRIS, K., THESSALOU-LEGAKI, M., PETRAKIS, G., \& CONIDES, A., 2010. Ontogenetic shifts and temporal changes in the trophic patterns of the deep-sea red shrimp, Aristaeomorpha foliacea (Decapods: Aristeidae), in the Eastern Ionian Sea (Eastern Mediterranean). Marine Ecology, 31(2), 341:354.

KATSANEVAKIS, S. 2008. Marine debris, a growing problem: Sources, distribution, composition, and impacts. In: HOFER TN (ed) Marine Pollution: New Research. Nova Science Publishers, New York. 53- 100

LAIST, D.W. 1997. Impacts of Marine Debris: Entanglement of Marine Life in Marine Debris Including a Comprehensive List of species with Entanglement and Ingestion Records. In: COE, J.M. \& ROGERS, D.B. (eds.). Marine Debris: Sources, Impacts and Solutions. Springer Series on Environmental Management, Springer-Verlag, New York. 99-139

LAIST, D.W., COE, J.M. \& O'HARA, K.J. 1999. Marine Debris Pollution. In: JOHN R. TWISS, JR. \& RANDALL R. REEVES (eds.). Conservation and management of marine mammlas.
Smithsonian Institution Press.

PEZZUTO, P. R., PEREZ, J.A. A. A., WAHRLICH, R. 2006. Deepsea shrimps (Decapoda: Aristeidae): new targets of the deepwater trawling fishery in Brazil. Braz. J. Oceanogr. 54(2/3):123134

PRUTER, A.T.. 1987. Sources, quantities and distribution of persistent plastics in the marine environment. Mar. Pollut. Bull. 18(6-B):305-310

REZENDE, G. A., 2011. Estudo da maturação sexual microscópica e dieta do camarão-carabineiro Aristaeopsis edwardsiana (Johnson, 1867) (Decapoda: Aristeidae) na região Sudeste-Sul do Brasil. Dissertação de Mestrado. Universidade Federal de Rio Grande, $68 \mathrm{p}$

RIBIC, C.A. \& GANIO, L.. 1996. Power analysis for beach surveys of marine debris. Mar. Pollut. Bull. 32(7):554-557.

ROTHSTEIN, S.I.. 1973. Plastic particle pollution of the surface of the Atlantic Ocean: evidence from a seabird. Condor 75: 344345

RYAN, P.G.. 1988. Effects of ingested plastic on seabird feeding: evidence from chickens. Mar. Pollut. Bull. 19: 125-128.

THOMPSON, R.C., OLSEN, Y., MITCHELL, R.P., DAVIS, A., ROWLAND, S.J., JOHN, A.W.G., MCGONIGLE, D. \& RUSSELL, A.E., 2004. Lost at sea: Where is all the plastic? Science 304: 838.

WIECZOREK, S.K., CAMPAGNUOLA, S., MOORE, P.G., FROGLIA, C., ATKINSON, R.J.A., GRAMITTO, E.M., \& BAILEY, N., 1999. Final Report: The composition and fate of discards from Nephrops trawling in Scottish and Italian Waters. EC Study Contract, 96/092.

ZITKO, V. \& HANLON, M.. 1991. Another source of pollution by plastics: skin cleaners with plastic scrubbers. Mar. Pollut. Bull. 22: $41-42$.

Submetido: 05/05/2011

Aceito: 07/12/2011 
